\title{
Planung und Vorbereitung bei Eingriffen im Bereich des proximalen Oberschenkels
}

\author{
Felix Bonnaire, Thomas Lein, Carsten Straßberger
}

\section{Zusammenfassung}

Operationen am proximalen Oberschenkel haben unterschiedliche Indikationen und Dringlichkeiten. Bei den Frakturen ist nur wenig Zeit für die Planung, bei degenerativen Erkrankungen können differenziertere Planungen vorgenommen werden. Das Ziel der Operation beeinflusst natürlich auch die Vorbereitung. Bei Frakturen sind die Ziele immer die möglichst anatomische Wiederherstellung der Achsenverhältnisse über einen voll belastbaren Kraftträger. Bei Korrekturosteosynthesen ist die erwünschte Korrektur das Hauptziel und bei der Endoprothetik eine möglichst komplikationsarme Implantation einer für den Patienten passenden und lange tragbaren Endoprothese. Manche Eingriffe sind vital indiziert, manche können ad libidum festgelegt werden. Diese Gesichtspunkte bestimmen die Vorbereitung des Patienten von der Aufklärung, den notwendigen Voruntersuchungen, der Risikoreduzie- rung im Vorfeld bis hin zur Lagerung des Patienten auf dem Operationstisch. Für die Lagerung ist allein entscheidend, dass der Operateur sich sicher sein kann, dass er mit ihr das gewünschte Operationsziel ohne Gefährdung der Asepsis optimal erreichen kann. Zudem müssen bestimmte „Operationsschulen“ berücksichtigt werden, die auf guten Erfahrungswerten über Jahrzehnte basieren.

\section{Planning and Preparation of Operations in the Region of the Proximal Thigh}

Operations on the proximal thigh have different indications and degrees of urgency. In cases of fractures there is very little time for planning, in cases of degenerative diseases a differentiated planning can be undertaken. The objective of the operation also has an impact on the preparation. In cases of fractures the aim is always to achieve the best possible anatomic reconstruction of the axis relationships over a fully loadable stress bearer. In the case of a correction osteosynthesis the main objective is the desired correction and for joint arthroplasties the implantation of an appropriate and long-lasting endoprosthesis with as few complications for the patient as possible. Some operations have vital indications, some can be chosen ad libidum. These points of view determine the preparation of the patient from the necessary explanations, the required preliminary examinations, the preoperative risk reduction through to the positioning of the patient on the operation table. Decisive for the positioning is solely the possibility for the surgeon to optimally achieve the desired surgical result without endangering the sterile conditions. In addition, specific "operative schools" that have been defined on the basis of good experience over many years must be taken into account.

\section{Einleitung}

Am proximalen Femur sind jährlich alleine wegen Frakturen etwa 100000 Operationen notwendig. Hinzu kommen etwa 150000 Hüftgelenkersatzoperationen in Deutschland pro Jahr. Die Zahl der $\mathrm{zu}$ erwartenden Operationen wird für die nächsten Jahre als kontinuierlich steigend vorausgesagt. Im Qualitätsreport 2009 des Aqua-Instituts werden die Gesamtergebnisse für die hüftgelenksnahen Frakturen mit einer Reoperationsrate von 3,2\% und für die Endoprothesenerstimplantation von $1,9 \%$ für den stationären Aufenthalt angegeben.

OP-JOURNAL 2011; 27: 62-69

(C) Georg Thieme Verlag KG Stuttgart · New York DOI http://dx.doi.org/10.1055/s-0030-1271034
Dabei ist die endoprothetische Versorgung bei Frakturen mit einer höheren Infektionsrate als bei den elektiven Hüftendoprothesenerstimplantationen behaftet. Die Spanne der Komplikationsraten in den einzelnen Kliniken ist enorm. Schon anhand dieser Aussagen und Zahlen ist es leicht nachvollziehbar, dass Komplikationen durch gute präoperative Planung und chirurgisches Management kontinuierlich verringert werden müssen (Qualitätsreport 2009 Aqua-Institut, www.aqua-institut.de).

\section{Allgemeine Vorbereitungen}

Alle Eingriffe am proximalen Oberschenkel und Hüftgelenk unterliegen therapeutischen Grundsätzen, die zum Großteil auch in den Leitlinien festgelegt sind. Grundsätzlich ist bei diesen Eingriffen die Thromboemboliegefahr hoch, auch das Risiko einer lokalen Komplikation wie einer Hämatombildung und Wundinfektion. Auch Gefäß- und Nervenschäden kommen in etwa $0,1-0,4 \%$ vor. Hauptgrund für Reoperationen am Knochen sind Repositionsverluste und Cut-outs bei den Osteosynthesen und Luxationen und Infektionen bei den Prothesen. Andere Risiken liegen in Dekubitalulzerationen, Harnwegsinfekten und Pneumonien bei 3,1\%. Zudem sind diese Verletzungen und Operationen alle mit einem nennenswerten Blutverlust verbunden, der möglicherweise zu perioperativen Substitutionen führt. Vor allem bei dringlichen Indikationen ist die Vorbereitungszeit kurz, eine Optimierung der Nebenerkrankungen wie Diabetes, Hypertonie, kardiovaskulären Erkrankungen, der peripheren arteriellen Verschlusskrankheit 

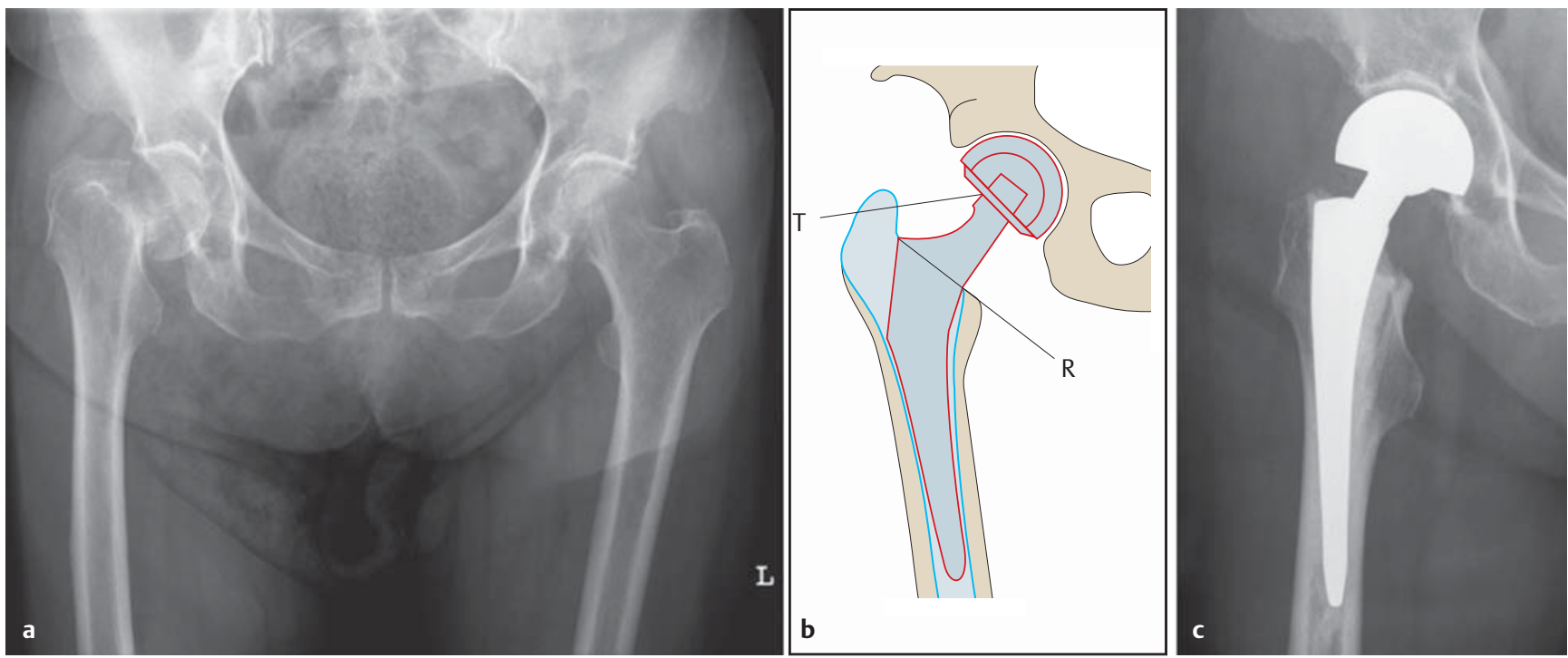

Abb. 1 a bis c Manuelle Planung zur Implantation einer Duokopfprothese (b) bei medialer Schenkelhalsfraktur rechts (a), definitive Versorgung (c).

mit deren lokalen Konsequenzen kann aus Zeitgründen nicht immer abgewartet werden.

Bei allen Eingriffen wird aus diesem Grunde

- eine risikoadaptierte Thromboseprophylaxe,

- eine Antibiotikaprophylaxe mit einem Cephalosporin der 2. Generation,

- eine Frühmobilisation und

- eine kurze intensive Zeit der präoperativen Vorbereitung mit interdisziplinärem Ansatz gefordert.

Die Blutgerinnung muss abgeklärt werden und gerinnungshemmende Medikamente für die Operation unter Umständen langfristig umgestellt werden. In der Regel werden 2-4 Blutkonserven bereitgestellt, wegen der Länge des Eingriffs wird

- ein Harnblasenkatheter vorbereitet.

- Eventuelle Unverträglichkeiten oder Allergien sollten berücksichtigt werden.

Bei elektiven Operationen sollten diese Risiken schon im Vorfeld mit dem Patienten abgeklärt werden [5]. Er sollte auf die Möglichkeit einer Eigenblutspende hingewiesen werden und mit den Risiken der vorgesehenen Operationen vertraut gemacht werden. Wichtige Vorbefunde sollten noch vor stationärer Aufnahme zusammengetragen und der Anästhesie zur Vorbereitung der Narkose zur Verfügung gestellt werden. Die benötigten Implantate sollten für den Eingriff bekannt sein und rechtzeitig (1 Tag vor dem Eingriff spätestens) zur Verfügung stehen.
Insbesondere sollte darauf geachtet werden, dass die Bildinformationen für die geplante Operation ausreichend sind, d.h., dass die anatomischen Abschnitte, die durch die Operation und das Implantat im weitesten Sinne verändert werden, in ausreichender Bildqualität und -übersicht dargestellt sind. Wesentliche Zusatzinformationen, die durch CT-Untersuchungen oder MRT-Untersuchungen gewonnen wurden, sollten ins elektronische Bildsystem (PACS) gestellt sein, damit man sie auch intraoperativ jederzeit aufrufen kann.

Bei Frakturen, deren Versorgung als Eingriffe von hoher Dringlichkeit gelten, hat man weniger Zeit zur Vorbereitung. Neben den allgemeinen Maßnahmen für alle Hüfteingriffe muss die allgemeine Operabilität festgestellt werden. Für manche Patienten, die in schlechtem $\mathrm{Zu}$ stand zur Aufnahme kommen (dekompensierte Herzinsuffizienz, schlecht eingestellter Diabetes, Exsikkose, Anämie oder Elektrolytstörung) kann eine kurze Vorbereitungszeit von 12-24h lebensrettend sein. Die Mehrheit der Patienten profitiert allerdings von der sofortigen Operation. Die Operation stellt eine wirksame Schmerztherapie dar, sie ist die Voraussetzung für die Mobilisation und letztere ist die Voraussetzung für die Vermeidung von Dekubitalulzera, Thrombosen und Embolien sowie Pneumonien.

Viele Patienten haben eine Dauermedikation mit Thrombozytenaggregationshemmern oder Warfarin. Thrombozytenaggregationshemmer sollten nicht abgesetzt werden, weil dann eine hohe
Stentverschlussrate v.a. im 1. Jahr nach Implantation droht. Zudem müsste man 7 Tage bis zur Normalisierung der Gerinnung warten - ein Risiko, welches wegen der signifikant erhöhten Mortalität nicht eingegangen werden darf. Die Komplikationsrate bez. Hämatomen und Blutungen ist nicht signifikant erhöht. Bei Gabe von Warfarinen (Marcumar und Falitrom) sollte der Grund für die Gerinnungshemmung mit dem Fachkollegen des indizierenden Faches (Angiologie, Kardiologie) diskutiert werden. Grundsätzlich ist eine sofortige Antagonisierung mit PPSB oder eine langsamere mit Vitamin $\mathrm{K}$ möglich. Risiken der Antagonisierung sollten gegen die Risiken der Operationsverschiebung abgewogen werden. In einer italienischen Studie konnte gezeigt werden, dass die Blutung unter Warfarin bei $\mathrm{Zu}$ warten auf die Operation zu erhöhten Raten von Bluttransfusionen geführt hat, weil die Patienten ohne Frakturversorgung in den Frakturbereich bluten. Wenn man mehr als 5 Tage auf die Operation gewartet hatte, zeigte sich die Mortalität signifikant erhöht. In dieser Studie war nach Gabe von 1-2,5 mg Vitamin K i.v. bei den meisten Patienten der QuickWert innerhalb von 24 Stunden normalisiert [4]. Derzeit ist eine PPSB-Gabe präoder intraoperativ je nach intraoperativer Blutungsneigung die beste Lösung. Eine zusätzliche Möglichkeit, Blut einzusparen, stellt die Wiederaufarbeitung des intraoperativen Blutverlusts mittels Cell-Saver dar. Dieser stellt auch eine Alternative bei geplanten Eingriffen und Ablehnung einer Eigenblutspende und Fremdbluttransfusion dar. 
Nicht wenige der Patienten leiden unter demenziellen Störungen. Dies kann zur Beeinträchtigung der Einwilligungsfähigkeit zur Operation führen. Spezielle Ausführungen hierzu siehe weiter unten im Text.

\section{Spezielle Indikationen}

\section{Elektivoperationen}

Endoprothetik

Die Vorbereitungen für Elektivoperationen unterscheiden sich wesentlich von denen bei Operationen hoher Dringlichkeit. Kompromisse bei der Befunderhebung sind nicht gerechtfertigt. Begleitende, optimierbare Erkrankungen sollten im Vorfeld optimal eingestellt werden. Die Bedürfnisse des Patienten sollten in hohem Maß berücksichtigt und mit den gegebenen Möglichkeiten abgeglichen werden. In der Regel wünscht sich der Patient Informationen über die Art der Prothese und ihre speziellen Eigenschaften, wie sie eingesetzt wird und wie lange sie voraussichtlich halten wird. Vor- und Nachteile gegenüber anderen Modellen möchte er kennenlernen, ebenso die Art und Weise der Nachbehandlung. Das Implantatdesign wie Oberflächenendoprothese, Kurzschaft oder Standardschaft und die Verankerung zementiert oder zementfrei ergibt sich in der Regel aus biologischem Alter, dem funktionellen Anspruch und den gegebenen Knochenverhältnissen. Spezielle anatomische Gegebenheiten oder das Vorhandensein der bereits erwähnten Allergieproblematik erfordern ein erweitertes Endoprothesenportfolio mit nickelfreien, anatomischen und Revisionsendoprothesen. Die Eigenblutspende und die alternative Blutaufarbeitung wurden bereits erwähnt.

Die Vorbereitung des Operateurs für die Operation beinhaltet die spezielle Anamnese des Patienten bez. seiner Symptome und die Funktionsdiagnostik des Gelenks. Anhand der bildgebenden Verfahren, die in der Regel eine tief eingestellte Beckenübersichtsaufnahme im Stehen und eine axiale Darstellung des proximalen Oberschenkels beinhalten, kann eine Analyse der Pathologie und der sich daraus ergebenden Maßnahmen erfolgen. Für die manuelle oder eine CAD-gestützte präoperative Implantatplanung ist die Anfertigung eines maßstabsgerechten Röntgenbilds unerlässlich. Gewährleistet wird dies durch einen standardisierten Filmfokusabstand und eine Kalibrierung

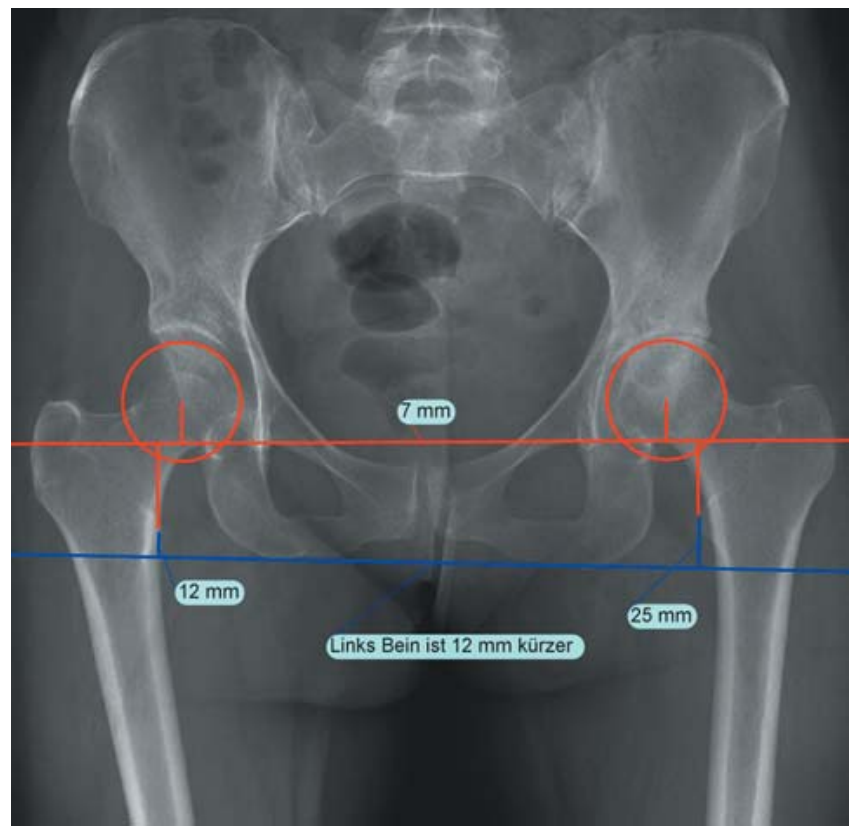

Abb. 2 Digitale Planung der funktionellen Anatomie mit Beinlängendifferenz (links $-12 \mathrm{~mm}$ ) und Markierung des anatomischen Drehzentrums links.
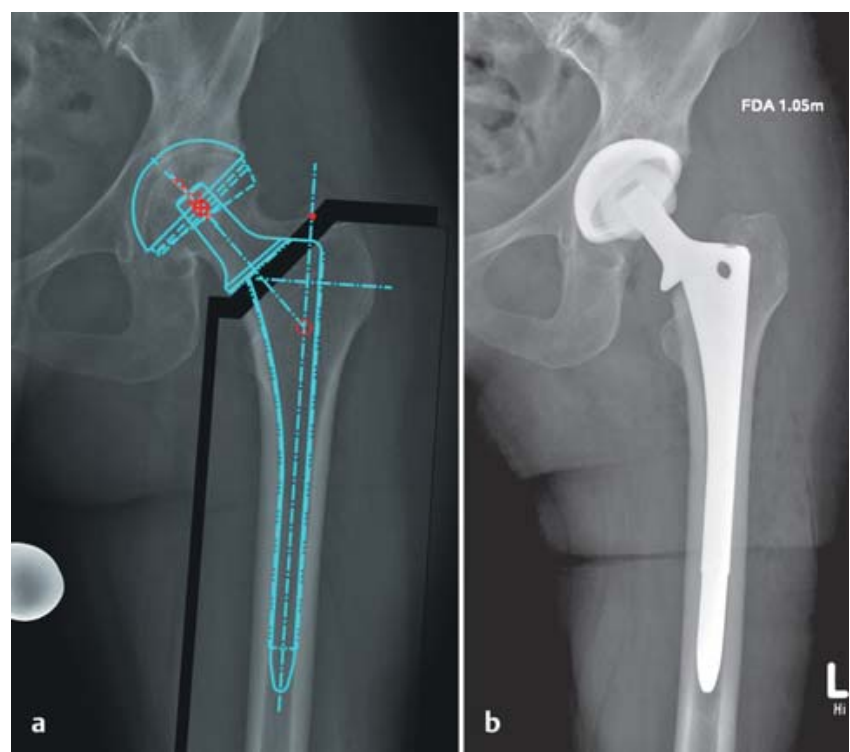

Abb. $3 \mathrm{a}$ und $\mathbf{b}$ Digitale Planung der Implantatpositionierung und Größe (a) sowie die postoperative Röntgenkontrolle (b).

des Röntgenbilds durch die Abbildung einer Metallkugel bekannten Durchmessers oder eines Zentimetermaßes.

Das Ziel der Endoprothesenimplantation ist eine möglichst gute Imitation der Anatomie der Gegenseite, falls diese gesund ist. Das heißt, dass die Längenund Winkelverhältnisse am proximalen Femur, einschließlich des Drehzentrums, verglichen werden. Die Knochenqualität, die Neigung und Überdachung des Oberschenkelkopfs, die Resektionslinie am Schenkelhals und die Weite der Markhöhle, ihre Konfiguration und die Kortikalisdicke muss der Operateur präoperativ erfassen. Auch Besonderheiten wie Varus- oder Valgusfehlstellungen mit
Veränderung des CCD-Winkels und verstärkte Antetorsion sowie Exophytenbildung sind wesentliche Vorinformationen für den Operateur.

\section{Planung}

Die Leitlinien in Orthopädie und Unfallchirurgie gehen von einer Planung der Endoprothesenimplantation in irgendeiner Form, meistens mittels maßstabsgerechter Schablonen oder entsprechend kalibrierter Software aus. Mittels Übertragung der anatomischen Verhältnisse der gesunden Seite durch Spiegelung auf die kranke Seite kann anhand von Zeichnungen auf durchscheinendem Papier die Wunschposition für die einzubringende Prothese imitiert werden. 


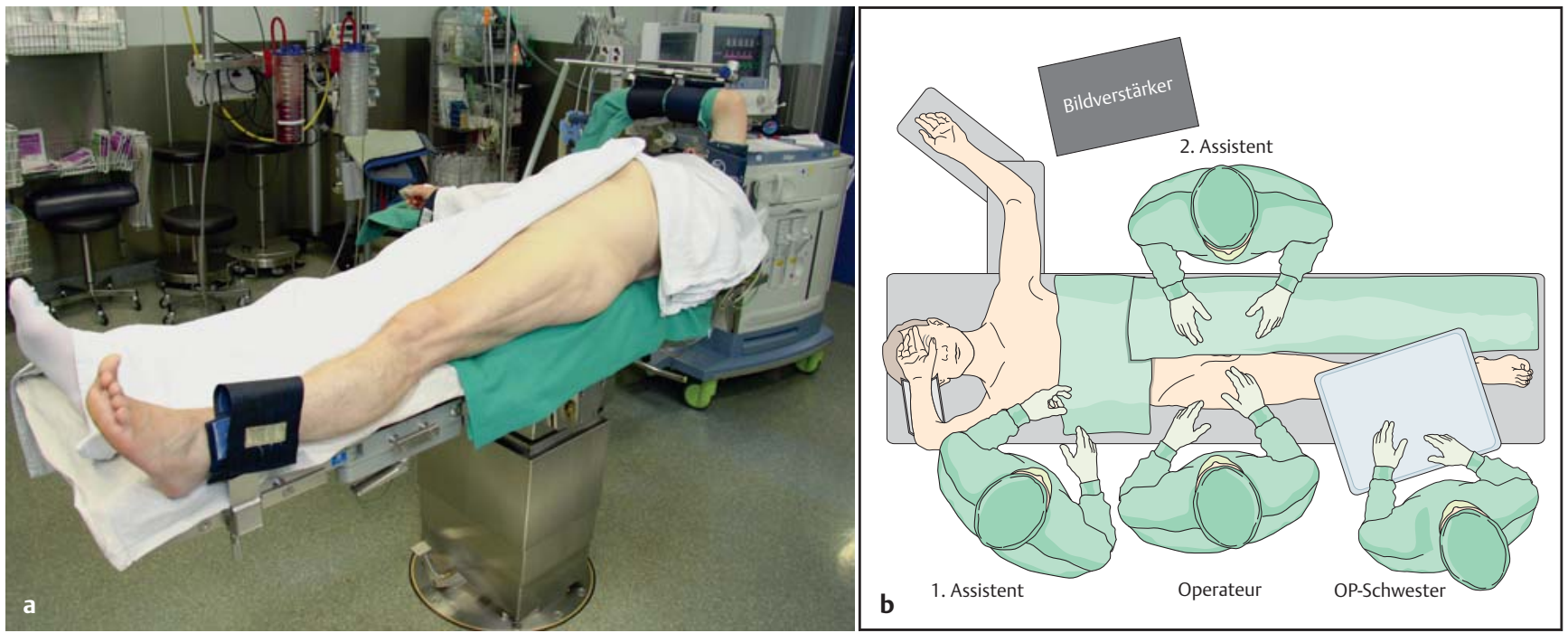

Abb. 4 a und b Positionierung in Rückenlage lateralisiert auf dem OP-Tisch (a). Schematische Darstellung zur Anordnung OP-Personal und Bildverstärker in Rückenlagerung (b).
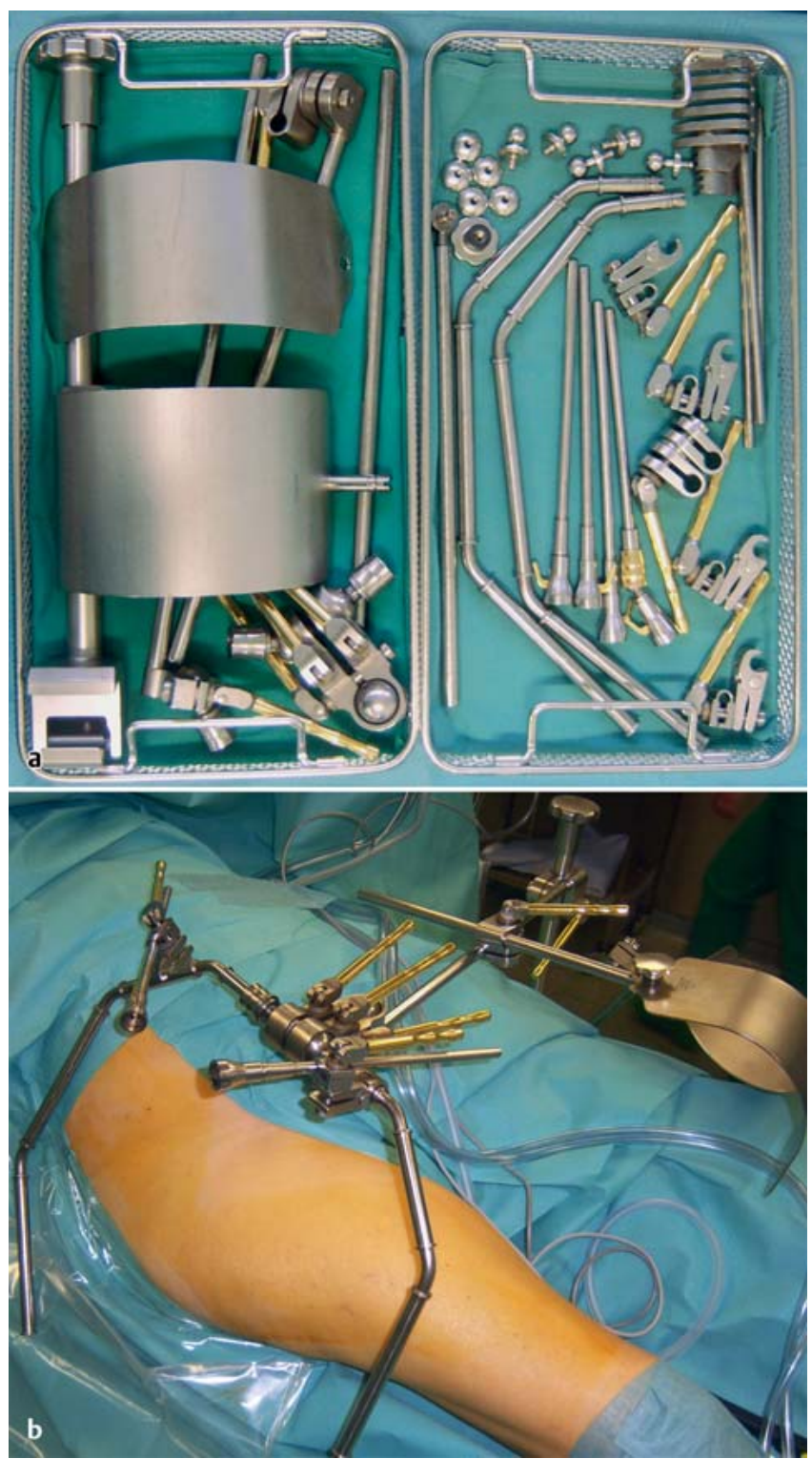

Abb. 5 a und $\mathbf{b}$ Siebinstrumentarium Hakensystem (a), montiertes Hakensystem bei Zugang zur Hüftgelenkspfanne (b).
In der Regel können so die Pfannengröße, Schaftdesign und Größe sowie die Resektionslinie am Schenkelhals gut festgelegt werden.

IT-unterstützte Zeichnungssoftware ermöglicht heute die Planung der Optimalsituation unter Berücksichtigung von nahezu fast allen Prothesenmodellen. Natürlich können die Optimalzeichnungen nicht immer operativ umgesetzt werden. Trotzdem ist es für den Operateur eine sehr gute Vorbereitung, sich mit den Gegebenheiten und den zu erstrebenden Veränderungen durch die Prothesen auseinanderzusetzen und möglichst nahe ans Ziel heranzukommen (Abb. 2 und 3).

\section{Zugang und Lagerung}

Der Zugang zur Hüfte bestimmt die Lagerung. Der Zugang wiederum wird von verschiedenen Operationsschulen gepflegt, die gute Gründe für das gewählte Vorgehen erarbeitet haben. Der häufigste Zugang ist der transmuskuläre laterale Zugang nach Bauer, gefolgt vom WatsonJones-Zugang und dem sog. hinteren $\mathrm{Zu}$ gang, jeweils modifiziert in minimalinvasiver Technik möglich. Die Lagerung für den Zugang nach Bauer und den Watson-Jones-Zugang ist die Rückenlage auf einem für die Durchleuchtung zugänglichen Tisch. Der Patient liegt dabei exzentrisch zur Seite der zu operierenden Extremität so, dass die Weichteile geringfügig über die Tischkante hängen (Abb.4). Die Lagerung erfolgt auf einer rutschfesten Unterlage, Röntgenschutzvorkehrungen sind in der Regel störend. Die Abdeckung erfolgt beweglich, wobei

Felix Bonnaire et al.: Planung und Vorbereitung bei Eingriffen im Bereich des proximalen Oberschenkels 
besonders darauf $\mathrm{zu}$ achten ist, dass die Einmal-U-Tücher auf trockenem Untergrund weit dorsal angeheftet werden, weil sie sich sonst frühzeitig lösen. Die Abdeckung darf die Operation auf keinen Fall beeinträchtigen, auch nicht die Beweglichkeit des Beines, welche für die Resektion des Schenkelhalses und die Manipulation an der Pfanne notwendig ist.

Navigationssysteme, die für die Hüftendoprothetik eingeplant werden, bedürfen einer speziellen Lagerung des Patienten, die auf das jeweilige System abgestimmt sein muss.

Eine sinnvolle Ergänzung stellt der Einsatz von Retraktionssystemen zur Entlastung des Personaleinsatzes dar, v.a. am Wochenende und im Dienst. Diese sind mittlerweile von verschiedenen Herstellern mit vielfältigen modularen Optionen erhältlich (Abb.5). Bei entsprechendem Einsatz reduziert sich der zusätzliche Zeitaufwand durch den Aufbau des Systems auf ein Minimum. Der Zugang zur Hüftgelenkspfanne und der Zugang zum Schaft in Vierer- bzw. „Kampfposition“ wird durch spezielle Beinschalen ermöglicht. Ein Vorteil ergibt sich neben dem Ersatz des 2. Assistenten bei Operation in Rückenlage aus der dauerhaft statischen Hakenpositionierung ohne Verlust oder Verminderung des Sichtfelds im Laufe des Eingriffs (Abb. 6).

Der dorsale Zugang wird in kontralateraler Seitenlage durchgeführt. Der Patient liegt exzentrisch mit den Weichteilen abschließend an der dem Operateur zugewandten Tischkante (Abb. 7). Röntgenkontrollen in der axialen Ebene sind erschwert, wenn überhaupt möglich.

\section{Metallentfernung}

Für Metallentfernungen am proximalen Femur bietet sich die Seitenlage an. Das zu operierende Bein wird beweglich abgedeckt und v.a. nach kranial genügend Platz belassen. Das zu operierende Bein wird leicht flektiert, wodurch sich die Weichteile anspannen (Abb. 7). Speziell die Nagelentfernung kann ein größeres Problem darstellen. Der Zugang erfolgt in der Regel über der vorgegebenen Narbe bis zur Faszie, die Faszie wird eröffnet und von der Muskelfaszie am M. gluteus medius oder am M. vastus lateralis gelöst und über ein Auseinanderhalten der Muskelanteile des M. gluteus medius kann die Trochanterspitze gut erreicht

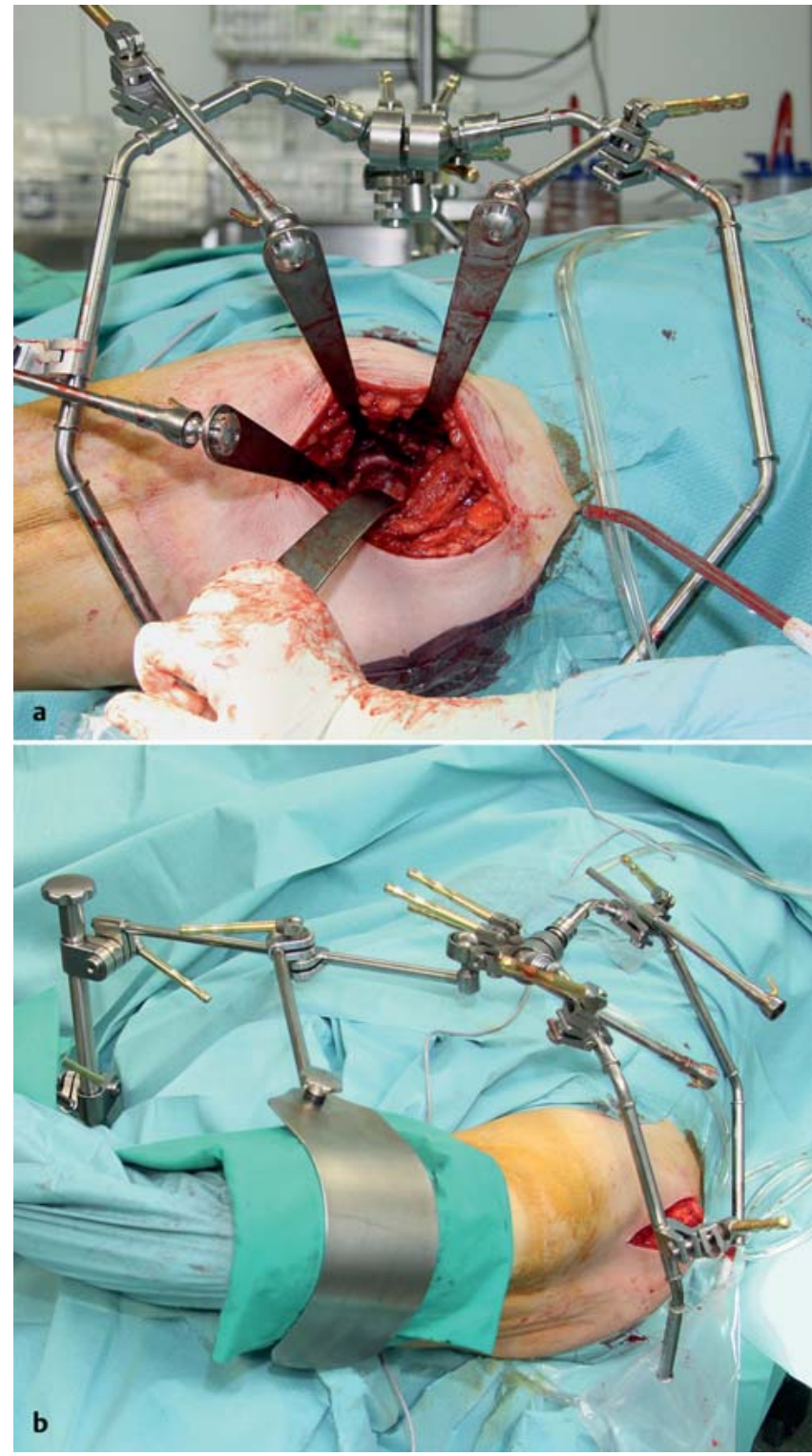

Abb. $6 a$ und $b$

Zugang zur Hüftgelenkspfanne unter Einsatz des Rahmenhakensystems (a). Positionierung des Beines bei Zugang zum Femurschaft in Viererposition (b).

werden. Die Darstellung der Verschlusskappe und des Nagels muss sehr sorgfältig erfolgen, um keine zusätzlichen Schäden in Muskulatur und Weichteilen, aber auch am Knochen zu setzen. Durch Flexion des Beines gelangt man in der Regel leicht in die Verlängerung des Markraums und damit in die Achse des Nagels. Durch zusätzliche Rotation am Bein kann der Zugang erleichtert werden. Der Zugang in Seitenlage ist auch für die Metallentfernung von Plattensystemen am proximalen Oberschenkel gut geeignet. Die Weichteile fallen nach Inzision der Haut und der Faszie leichter auseinander, durch Innen- und Außendrehen des Unterschenkels gelangt man leichter als in Rückenlage zum Implantat. Die Röntgendarstellung von abgebrochenen Implantaten kann jedoch durch diese Lage kompromittiert werden. Vor der Metall- entfernung sollte man sich immer vergewissern, dass der ehemalige Bruch auch sicher geheilt ist. Gerade bei Schenkelhalsfrakturen kann man sich leicht täuschen. Eine präoperative CT kann nur empfohlen werden.

\section{Korrekturoperationen}

Die Vorteile der Seitenlage können ohne Einschränkung auf Reeingriffe an der Hüfte übertragen werden, auch wenn man die Nachteile beim Röntgen benennen muss. Der Grund für den Vorteil liegt in der leichteren Darstellbarkeit einer anatomischen Situation bzw. Zugangsfläche, die keine sehr hohe Instabilität beinhaltet. Die Manipulation in Seitenlage gelingt in der Regel leichter als in Rückenlage, v.a. stören nicht die dorsalen Weichteile. Die zur Operation füh- 


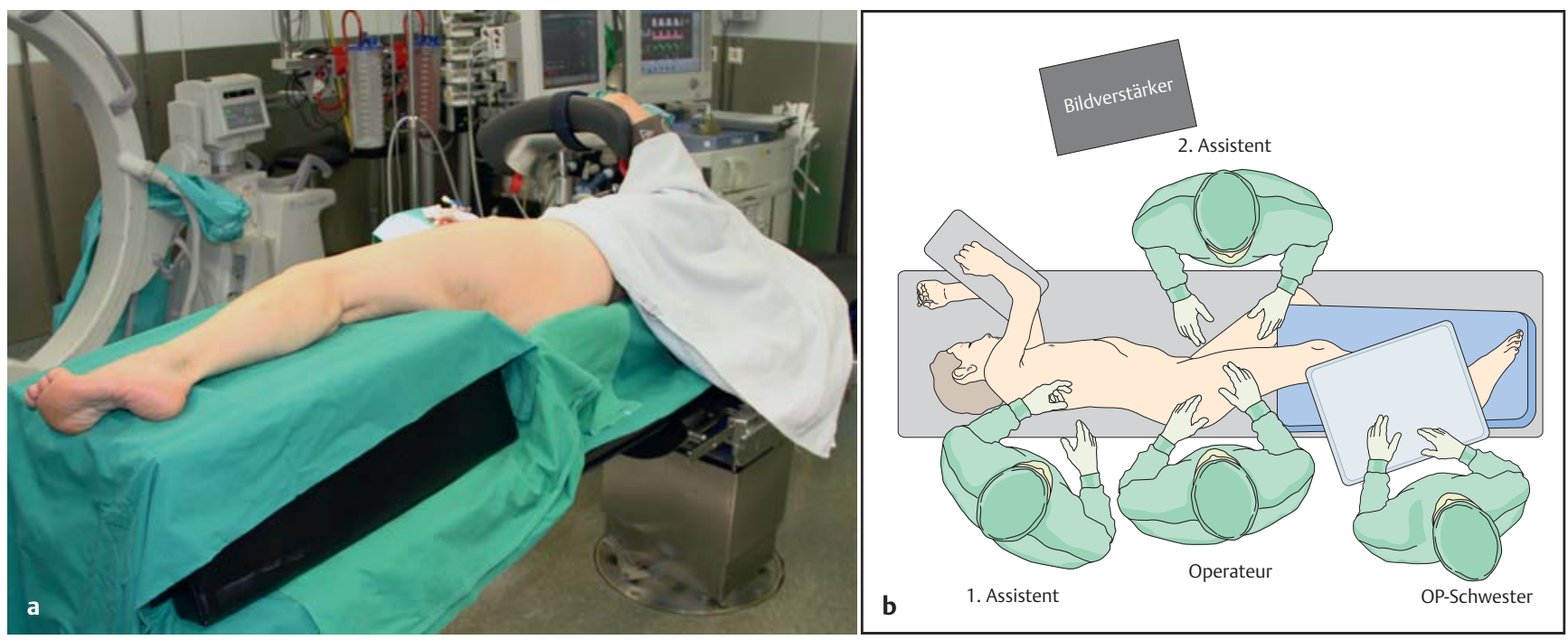

Abb. 7 a und b Seitenlagerung für Zugang zu Femur und Hüftgelenk (a). Schematische Anordnung zur Positionierung OP-Personal und Bildverstärker in Seitenlagerung (b).
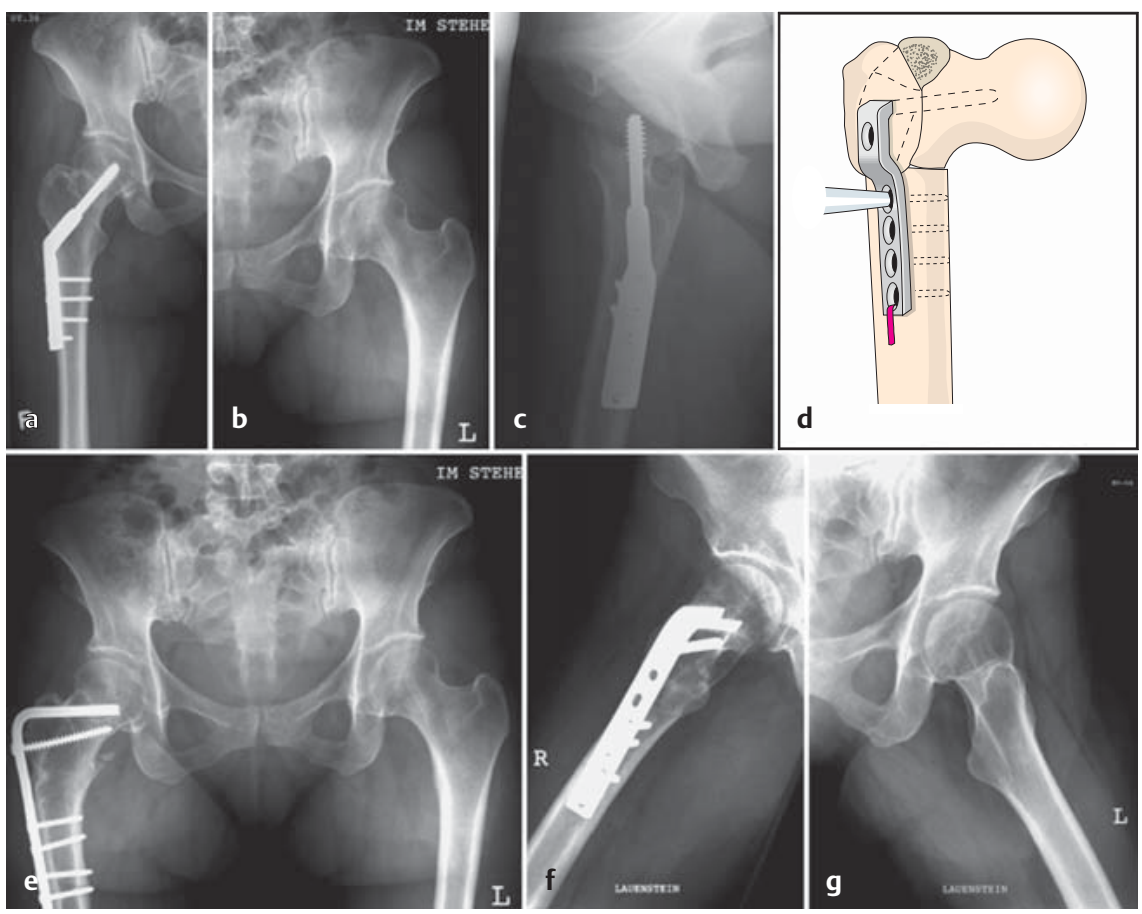

Abb. 8 a bis $\mathbf{g}$ Beispiel: 34-jährige Patientin mit Schenkelhalsfraktur: Fixation und Heilung in Valgusposition, Retroversion des Kopfes und Außenrotation des Femurs (a und $\mathbf{b}$ ). Gegenseite (c). Korrekturzeichnung mit Operationsprinzip (d). Erreichte Korrektur im Stand (e). Vergleich der Achse rechts und links nach Ausheilung der Korrektur ( $\mathbf{f}$ und $\mathbf{g}$ ).

rende Fehlstellung muss klar identifiziert sein. Die Achsenverhältnisse sollten mit der gesunden Gegenseite verglichen werden. Ein Planungs-CT kann hilfreich sein. Bei Korrekturoperationen wegen Heilungsstörung am Schenkelhals empfiehlt sich eine MRT-Untersuchung zur Darstellung der Perfusionsverhältnisse. Die erwünschten Korrekturen können mit einer Planungszeichnung oder -software im Vergleich zur gesunden Gegenseite eingezeichnet werden (Abb. 8).

\section{Frakturen}

Hüftgelenksnahe Frakturen haben besondere Eigenheiten. Aufgrund der erhöhten Komplikationsrate bei längerem Zuwarten auf die Operation (48 Stunden) ist eine hohe Dringlichkeit geboten, die andere Vorgehensweisen und manche Kompromisse einfordert [3,6,9-12]. Weiterhin verbessert die rasche operative Versorgung nachweislich das Ergebnis in Bezug auf Wiedererreichen der per- sönlichen Selbstständigkeit nach stattgehabter Schenkelhalsfraktur [1]. Betroffen sind immer mehr ältere Patienten mit vielen Nebenerkrankungen aus Seniorenheimen und allein wohnende ältere Menschen. Die Anamnese ist bei diesen Patienten nicht immer einfach durchzuführen, für die Fremdanamnese steht nicht immer ausreichend Zeit zur Verfügung. Die Umstände des Unfalls wie Unfallhergang oder die Unfallzeit sind nicht immer genau bekannt. Die Patienten sind unter Umständen schon vor dem Unfall dement oder zeitweise verwirrt. Ihre Medikation ist nicht immer bekannt, oft werden Gerinnungshemmer eingenommen. Durch die Schmerzen und den zusätzlichen Blutverlust sind zusätzlich vorübergehende Verwirrtheitszustände häufig. Wichtigste Erstmaßnahme ist zunächst die Herbeiführung einer Analgesie bei diesen hochgradig schmerzhaften Verletzungen.

Schon vor der bildgebenden Diagnostik sollte bei eindeutiger klinischer Diagnose eine suffiziente Schmerztherapie beginnen. Diese kann i.v. oder mit peripheren Nervenblockaden erfolgen. Die Diagnostik sollte möglichst schmerzfrei durchgeführt werden können. Gleichzeitig sollte ein venöser Zugang zur präoperativen Labordiagnostik und zur Infusion einer Elektrolytlösung gelegt werden.

Die Diagnostik beinhaltet in der Regel eine tief eingestellte Beckenübersichtsaufnahme, verbunden mit einer Lauenstein-Aufnahme des Hüftgelenks und proximalen Oberschenkels. Bei pathologischen Frakturen sollte das gesamte Femur einschließlich des Kniegelenks 


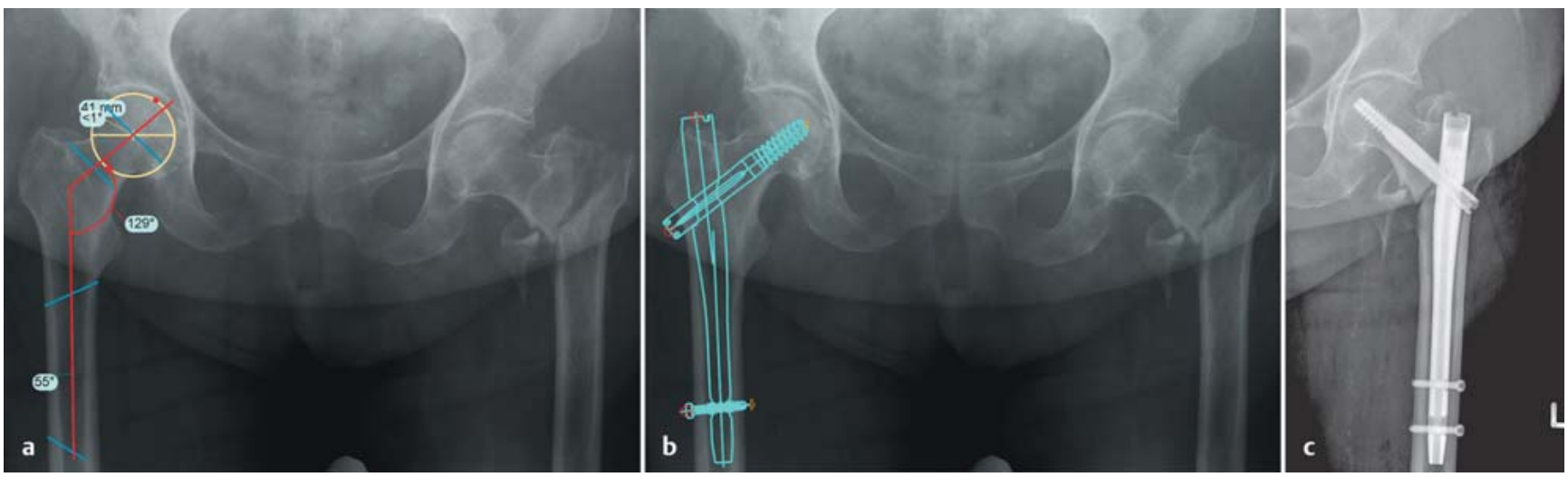

Abb. 9 a bis c Beckenübersicht bei pertrochantärer Femurfraktur links (31 A 2.2) und digitaler Planung der Achsenanatomie an der Gegenseite (a), digitale Planung Implantat an Gegenseite (b), definitive Versorgung a.-p. Aufnahme (c).

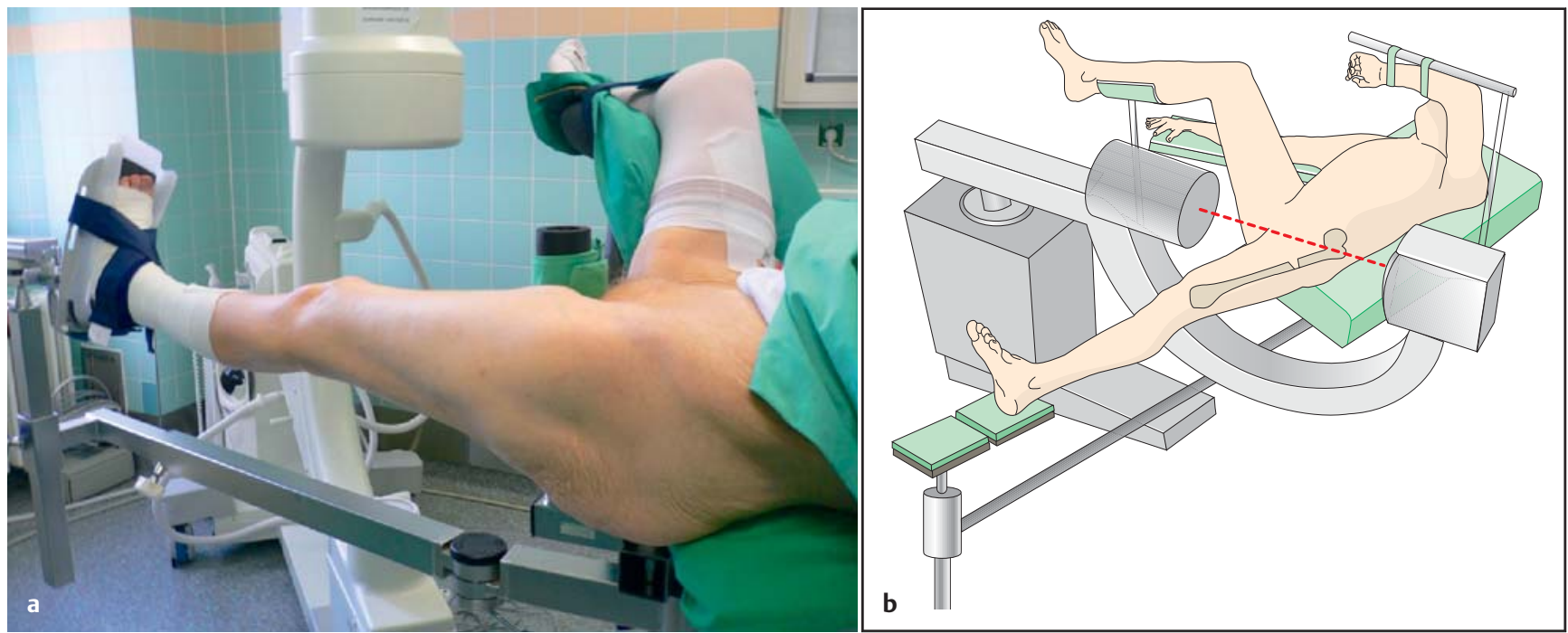

Abb. 10 a und b Lagerung auf dem Extensionstisch zur Versorgung einer Schenkelhalsfraktur (a). Schematische Darstellung zur Lagerung auf dem Extensionstisch (b).

dargestellt werden. Analog zu den endoprothetischen Eingriffen ermöglicht die softwaregestützte Planung eine präoperative Orientierung bez. des zum Einsatz kommenden Osteosyntheseimplantats. Die digitale Planung erfolgt ebenfalls an einem kalibrierten Röntgenbild anhand der Anatomie der nicht verletzten Seite. In speziellen Fällen wie periprothetischen Frakturen oder Frakturen des Schaftbereichs ohne wesentliche Rotationskomponente ist mit der digitalen Planung eine virtuelle Reposition der Fragmente und das Einpassen des ausgewählten Implantats möglich. Beachtet werden muss die Limitierung durch die 2-dimensionale Darstellung der Fraktur (Abb. 9).

Nach gesicherter Diagnose werden eine Thoraxaufnahme und ein EKG angefertigt sowie spätestens jetzt ein Anästhesist informiert.
In der Regel werden dann folgende Fragen aufgeworfen:

1. Ist der Patient in einem operablen Zustand und kann ohne weitere Maßnahmen operiert werden oder sind Maßnahmen zur Wiederherstellung der Operabilität Voraussetzung für die Operation?

2. Kann der Patient in die Operation einwilligen oder müssen andere Verantwortliche für die Einwilligung herbeigezogen werden?

3. Wie ist mit gerinnungshemmender Medikation umzugehen? Ist ein Zuwarten bis zur Wiederherstellung einer normalen Gerinnung gerechtfertigt oder schädigt es den Patienten?

4. Wann ist der nächste OP-Saal frei und welches Team steht zur Verfügung?

Ad. 1 Diese Frage ist in Abhängigkeit von der Anamnese der körperlichen Belastbarkeit, der Blutungsanamnese und der körperlichen Untersuchung in den meis- ten Fällen schnell zu klären. In der Regel wird eine Blutentnahme bei diesen Patienten empfohlen mit Bestimmung des kleinen Blutbilds, der Elektrolyte, der Retentions- und Leberwerte sowie der Gerinnung. Mit zunehmender Zahl der Laboruntersuchungen steigt die Wahrscheinlichkeit, zufällig außerhalb der Norm liegende Parameter zu finden, die nicht behandlungsrelevant sind.

Ad. 2 Bei Operationen wegen hüftnahen Frakturen handelt es sich um Eingriffe von hoher Dringlichkeit, weil eine zuwartende Haltung eine erhöhte Morbidität und Mortalität des Patienten nach sich zieht [3,6,9-12]. Menschen mit mentalen Störungen sind nicht immer in der Lage, Bedeutung und Tragweite des Eingriffs zu ermessen und ihre Entscheidung nach Beratung zu fällen. Für Patienten, für die kein Betreuer bestellt ist und die als einwilligungsfähig eingeschätzt werden, ergibt sich keine Proble- 
matik. Die Einwilligungsfähigkeit hat der Arzt zu beurteilen. Es gilt zwar ,je komplexer und risikoreicher der Eingriff, desto höher sind die Anforderungen an die Einwilligungsfähigkeit", andererseits dürfen die Ansprüche nicht übertrieben sein. Spricht aus der Sicht des Arztes nichts für ein Fehlen der Einwilligungsfähigkeit, so kann er ohne Weiteres davon ausgehen, dass sie vorliegt. Erweist sich später das Gegenteil als richtig, kann der Arzt nicht haftungs- oder strafrechtlich belangt werden. In Notfällen und Eilfällen hilft und rechtfertigt die $\mathrm{Ge}$ schäftsführung ohne Auftrag. Der Arzt hat die Behandlung durchzuführen, die dem mutmaßlichen Willen des Patienten entspricht.

Schwierigkeiten ergeben sich dann, wenn ein Betreuungsverhältnis eingerichtet ist. Verfügt der Patient über eine Einwilligungsfähigkeit, so ist allein seine Willensäußerung maßgeblich. Betreuung entmündigt nicht. Je länger allerdings der Eingriff hinausgeschoben wird, desto höher werden die Anforderungen an die Einwilligungsfähigkeit bzw. an die Einrichtung einer Betreuung [2].

Ad. 3 Siehe Text zu allgemeinen Vorbereitungen.

\section{Literatur}

${ }^{1}$ Al-Ani AN, Samuelsson B, Tidermark J et al. Early operation on patients with a hip fracture improved the ability to return to independent living. A prospective study of 850 patients. J Bone Joint Surg [Am] 2008; 90: 1436-1442

${ }^{2}$ Kern BR. Der betreute Patient - Betreuungsrecht. Ärzteblatt Sachsen 2008: 410-415

${ }^{3}$ Bottle A, Aylin P. Mortality associated with delay in operation after hip fracture: observational study. BMJ 2006; 332: 947-951

${ }^{4}$ Dettoni F, Castoldi F, Mauriziano U et al. Influence of timing and oral anticoagulant/antiplatelet therapy on outcomes of patients affected by hip fractures. Eur J Trauma Emerg Surg; in press

${ }^{5}$ DGA Info. Präoperative Evaluation erwachsener Patienten vor elektiven, nicht kardiochirurgischen Eingriffen. A \& I 2010; 51: S788S797

${ }^{6}$ Gdalevich M, Cohen D, Yosef D et al. Morbidity and mortality after hip fracture: the impact of operative delay. Arch Orthop Trauma Surg 2004; 124: 334-340

${ }^{7}$ Khan SK, Kalra S, Khanna A et al. Timing of surgery for hip fractures: a systematic review of 52 published studies involving 291, 413 patients. Injury 2009; 40: 692-697

${ }^{8}$ Kostuj T, Smektala R. Quality assurance using routine data. Is outcome quality now measurable? Unfallchirurg 2010; 113: 1047-1048, 1050-1052

${ }^{9}$ Moran CG, Wenn RT, Sikand M et al. Early mortality after hip fracture: is delay before surgery important? J Bone Joint Surg [Am] 2005; 87: 483-489
10 Shiga T, Wajima Z, Ohe Y. Is operative delay associated with increased mortality of hip fracture patients? Systematic review, metaanalysis, and meta-regression. Can J Anaesth 2008; 55: 146-154

11 Simunovic N, Devereaux PJ, Sprague $S$ et al. Effect of early surgery after hip fracture on mortality and complications: systematic review and meta-analysis. CMAJ 2010; 182: 1609-1616

12 Smektala R, Endres HG, Dasch B et al. The effect of time-to-surgery on outcome in elderly patients with proximal femoral fractures. BMC Musculoskelet Disord 2008; 9: 171

\section{Prof. Dr. med. Felix Bonnaire Chefarzt \\ Dr. med. Thomas Lein \\ Leitender Oberarzt \\ Dr. med. Carsten Straßberger \\ Facharzt}

Klinik für Unfall-, Wiederherstellungsund Handchirurgie

Städtisches Klinikum

Krankenhaus Dresden-Friedrichstadt

Friedrichstraße 41

01067 Dresden

unfallchirurgie@khdf.de 